\title{
Diseño e implementación de rúbricas como instrumento de evaluación del curso de Química General e inorgánica para estudiantes de ingeniería
}

\section{Resumen}

\author{
$\varnothing$ PEDRonel Araque-Marín ${ }^{1}$ \\ SANDRA ADELA TORIJANO-GUTIÉRREZ ${ }^{1}$ \\ NiCOLÁS ARANGO-LONDOÑO ${ }^{1}$
}

La evaluación no puede limitarse a una prueba centrada en la repetición de contenidos que pretende valorar el grado de aprendizaje de los estudiantes, la evaluación debe brindar otro horizonte con relación a la calificación tradicional, es decir debe ser una herramienta válida para el proceso de enseñanza-aprendizaje. En el presente trabajo se propuso un diseño de rúbrica como instrumento de evaluación para la asignatura de Química General e Inorgánica de la Universidad EIA. La rúbrica basada en objetivos de aprendizaje, aspectos a valorar, descriptores, escala de calificación y criterios, fue implementada durante los periodo académico 2015-2 y 2016-1, la propuesta facilitó la calificación de desempeño de los estudiantes de primer semestre de ingeniería, no solo sobre lo que aprendieron, sino también de cómo aprendieron y las competencias desarrolladas en el proceso evidenciando diferencias estadísticamente significativa ( $p$-valor $<0,05)$ en el número de estudiantes que aprobaron la asignatura.

Palabras claves: Rúbrica; Evaluación; Química General; Química Inorgánica; Enseñanza-aprendizaje; Ingeniería

\section{Design and implementation of rubrics as an instrument for the evaluation of General and Inorganic Chemistry course for engineering students}

\section{Abstract}

The assessment can not be limited to a test focused on the repetition of content that aims to assess the degree of student learning, assessment should provide another horizon in relation to the traditional rating, ie must be a valid tool

1 Universidad EIA, Envigado, Colombia.

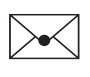

Autor de correspondencia: Araque Marín, P. (Pedronel): Universidad EIA, Sede de Las Palmas: Km 2 + 200 Vía al Aeropuerto José María Córdova, Envigado, Colombia. Código Postal: 055428. Teléfono: (574) 3549090 Opción 1, Ext. 234. Correo electrónico: pedronel.araque@eia.edu.co
Historia del artículo:

Artículo recibido: 17-I-2017/ Aprobado: 16-I-2019

Disponible online: 3 de febrero de 2019

Discusión abierta hasta octubre de 2020 
for teaching-learning process. In this paper we design a proposal as an evaluation tool for the subject of General and Inorganic Chemistry of the University EIA. the rubric based on learning objectives, aspects to be evaluated, descriptors, criteria and rating scale, was implemented during the 2015-2 and 2016-1 academic year, the proposal facilitated the performance rating of freshmen engineering, not only about what they learned, but also how they learned and skills developed in the process demonstrating statistically significant difference ( $p$-value $<0.05)$ in the number of students who passed the course.

Keywords: Rubric; Evaluation; General Chemistry; Inorganic Chemistry; Teaching-learning; Engineering

\section{Concepção e implementação de rubricas como instrumento de avaliação do curso de Química Geral e Inorgânica para estudantes de engenharia}

\section{Resumo}

A avaliação não pode ser limitado a um teste centrou-se na repetição de conteúdo que visa avaliar o grau de aprendizagem dos alunos, a avaliação deve fornecer outro horizonte em relação à classificação tradicional, ou seja, deve ser um instrumento válido para o processo de ensino -Learning. Neste projeto seção de papel proposto como uma ferramenta de avaliação para o objecto de Geral e Inorgânica da Universidade EIA. A posição baseada em objetivos de aprendizagem, aspectos a serem avaliados, descritores, critérios e escala de classificação, foi implementado durante o ano lectivo 20152 e 2016-1, a proposta facilitou a classificação da engenharia calouros desempenho, não só sobre o que aprenderam, mas também como eles aprenderam e habilidades desenvolvidas no processo demonstrando diferença estatisticamente significativa $(\mathrm{p}<0,05)$ no número de alunos que passaram o curso.

Palavras-chave: Rubrica; Avaliação; Química Geral; Química Inorgânica; Ensino-aprendizagem; Engenharia.

\section{Introducción}

La química es una ciencia experimental que transforma tanto sustancias como su propio lenguaje químico. Un lenguaje que es vital en los procesos de enseñanza-aprendizaje, especialmente para nombrar los objetos de estudio "sustancias" proporcionando un vocabulario conceptual acerca de las leyes, modelos y teorías que rigen el comportamiento de elementos, sustancias simples y sustancias compuestas (Jacob, 2001). El aprendizaje de la química no se produce de manera repentina, sino que se trata de un proceso que toma tiempo, dado que se promueve con mayor facilidad en la medida que el estudiante tenga encuentros con situaciones y contenidos similares de los que puede abstraer conceptos; por eso, el tiempo es un factor de gran importancia a la hora de planificar y desarrollar las actividades pertinentes (Araque, 2013). Entre los procesos de enseñanza-aprendizaje de la química es importante realizar tres reflexiones: la primera consiste en conocer cómo tiene estructurado el estudiante los conocimientos del tema, para planearle la construcción de conceptos adecuados con el fin de correlacionar su saber existente en la estructura cognitiva con los nuevos conocimientos aportados por las actividades propuestas en el aula de clase; la segunda consiste en proporcionar un verdadero aprendizaje significativo porque desconocerlo nos induce a entregar información inadecuada a los 
estudiantes suponiendo que "lo deben saber", pero sólo están copiando la información para memorizarla, reproducirla y finalmente olvidarla y la tercera tiene que ver con el proceso de evaluación formativa que facilite la valoración del desempeño de los estudiantes incrementando la objetividad de un área que ha sido considerada subjetiva y compleja.

La rúbrica se puede considerar como un conjunto de características de una prueba constituida por tareas (Bachman, Palmer, 1996), además se ha definido como un instrumento de evaluación (Mansoor, Grant, 2002) que especifica el desempeño esperado y los diversos niveles de competencia a los que los estudiantes pueden llegar en el desarrollo de una habilidad. Es considerada también para tareas evaluativas auténticas. El desarrollo de prácticas evaluativas justas y que sean además transparentes, siguiendo principios democráticos, es decir participativos e incluyentes; estos principios se volverían indispensables en los lineamientos a seguir en el diseño de procedimientos de un sistema de evaluación que garantice un impacto positivo en los procesos de enseñanza-aprendizaje, el cual se puede traducir en una evaluación para el aprendizaje y debe ser el principal objetivo que guíe todo evento evaluativo en el aula (Picón, 2013), esta metamorfosis trae modificaciones en el proceso de evaluación considerando que la evaluación no puede limitarse a un solo tipo de prueba centrada en la repetición de contenidos y que pretenda solo alcanzar una calificación (Cano, 2008). Un instrumento de evaluación basado en una escala cualitativa y/o cuantitativa asociada a criterios que puedan medir las acciones de los estudiantes sobre los aspectos de las actividades que serán evaluadas (Torres, Perera, 2010) y desde estos criterios, poder juzgar, valorar, calificar y conceptualizar sobre un determinado aspecto del proceso, existiendo una conexión entre la enseñanza y la evaluación, es decir debe existir una concordancia entre lo que se está enseñando y lo que se está midiendo (Williams, 2003). La escala holística presenta una descripción general de los diferentes niveles de logro esperado en el desempeño de los estudiantes respecto a una tarea determinada. La escala analítica describe cada uno de los elementos del marco definido como constructo para cada nivel de logro de la escala, siendo estas más efectivas en procesos de evaluación en el aula dado que posibilita una realimentación detallada, descriptiva y dialógica entre el profesor y el estudiante (Gipps, 1999).

En el presente artículo se diseñó e implementó una rúbrica del tipo analítica como instrumento de evaluación del curso de Química General e Inorgánica, del primer semestre de los programas de ingeniería de la universidad EIA. La experiencia nos proporcionó elementos necesarios para elaborar descripciones más detalladas a medida que se familiarizó con los diferentes niveles de desempeño de los estudiantes con el fin de aumentar la tasa de aprobación de la asignatura.

\section{Metodología}

El diseño e implementación de la rúbrica se desarrolló en cuatro fases como se plantean a continuación:

Fase de preparación de la estrategia docente

Se revisaron detalladamente contenidos del curso de Química General e Inorgánica con el fin de definir unos objetivos de aprendizaje apropiados y de esta forma identificar los criterios de evaluación y/o cualidades específicas observables en el proceso realizado por los estudiantes, estableciendo diferentes niveles y asignar un porcentaje de la calificación final (Malini, Andrade, 2010).

\section{Fase de diseño de la rúbrica}

Con el fin de evaluar el desempeño del estudiante y de esta forma desglosar sus componentes para obtener una calificación total, se utilizó una rúbrica tipo analítica (Gatica, Urribarren, 2013), dado que esta puede utilizarse para determinar el estado del desempeño, debilidades y permite que los estudiantes conozcan lo que requieren para mejorar (Shipman et al., 2012). Las rúbricas fueron diseñadas para el 
examen parcial y final de la asignatura Química General e Inorgánica, dado que el examen parcial contiene el 50\% del curso y el examen final el 50\% restante.

\section{Fase de construcción de las rúbricas}

Para la construcción de la rúbrica examen parcial se tuvieron en cuenta los siguientes contenidos: balanceo de ecuaciones, nomenclatura inorgánica, estequiometria y fórmula molecular. Para la rúbrica examen final se plantearon los contenidos: equilibrio químico no acuoso, equilibrio químico acuoso y electroquímica.

TABLA 1. NÚMERO DE ESTUDIANTES POR PROGRAMA DE INGENIERÍA

\begin{tabular}{l|c|c|}
\multirow{2}{*}{$\begin{array}{c}\text { Programa de } \\
\text { Ingeniería }\end{array}$} & \multicolumn{2}{|c}{ Número de estudiantes } \\
\cline { 2 - 3 } Administrativa & $2014-2 / 2015-1$ & $2015-2 / 2016-1$ \\
\hline Ambiental & 44 & 33 \\
\hline Biomédica & 32 & 9 \\
\hline Civil & 26 & 32 \\
\hline Sistemas y \\
Computación & 6 & 34 \\
\hline Financiera & 7 & 14 \\
\hline Geológica & 11 & 11 \\
\hline Industrial & 19 & 8 \\
\hline Mecánica & 13 & 16 \\
\hline Mecatrónica & 22 & 7 \\
\hline Total & 189 & 32 \\
\hline
\end{tabular}

\section{Fase de Implementación}

Las rúbricas fueron implementadas durante los periodos académicos 2014-2/2015-1 y 20152/2016-1 en la Tabla 1 se detallan el número de estudiantes por programa de ingeniería donde se aplicó el estudio. Se propuso como exámenes parcial y final una actividad de modo escrito y de forma individual en el curso de Química General e Inorgánica del primer semestre de ingeniería de la universidad EIA. Se consideraron en total 385 divididos en 12 grupos, cada grupo con aproximadamente 32 estudiantes y una distribución por género de 63\% hombres y $37 \%$ mujeres.

\section{Fase de seguimiento y análisis}

Se compararon las notas alcanzadas por los estudiantes con las dos formas de evaluar, la implementación de la rúbrica "nueva propuesta" con 196 estudiantes entre los periodos 2015-2 y 2016-1 y la calificación tradicional "clásica" con 189 estudiantes entre los periodos 2014-2 y 2015-1, generando el factor EVALUACIÓN con dos niveles. Los resultados se analizaron con el software STATGRAPHICS Centurion XVI versión 16.2.04.

\section{Resultados y Discusión}

\section{Objetivos de aprendizaje y criterios de evaluación}

Teniendo en cuenta el 50\% inicial de los contenidos del curso se lograron definir y resumir en las Tablas 2, 3 y $\mathbf{4}$ los objetivos de aprendizaje con sus respectivos criterios, para el diseño de la rúbrica como instrumento de evaluación, en la Tabla 2 se indican los elementos para el tema de ecuaciones químicas y nomenclatura inorgánica; en la Tabla 3 los elementos para el tema de estequiometría y en la Tabla 4 los elementos para el tema de fórmulas mínima y/o molecular.

\section{Elementos para la rúbrica de exa- men final}

Teniendo en cuenta el 50\% restante de los contenidos del curso, se lograron definir los objetivos de aprendizaje con sus respectivos criterios, en la Tabla 5 se resumen los elementos como objetivos de aprendizaje y criterios necesarios para el diseño de la rúbrica como instrumento de evaluación de los temas equilibrio químico no acuoso; en la Tabla 6 los elementos para el tema de equilibrio químico acuoso y en la Tabla 7 los elementos para el tema de electroquímica. 
TABLA 2. OBJETIVOS DE APRENDIZAJE Y CRITERIOS DE EVALUACIÓN PARA LOS TEMAS BALANCEO DE ECUACIONES QUIIMICAS Y NOMENCLATURA INORGÁNICA

\begin{tabular}{l|l}
\multicolumn{1}{c|}{ OBJETIVOS DE APRENDIZAJE } & \multicolumn{1}{c}{ CRITERIOS } \\
\hline $\begin{array}{l}\text { Proponer la estructura y el nombre de las sustancias } \\
\text { empleadas en una reacción química. }\end{array}$ & $\begin{array}{l}\text { Interpreta adecuadamente el nombre de las sustancias } \\
\text { inorgánicas. }\end{array}$ \\
\hline $\begin{array}{l}\text { Establecer la conservación de elementos en una ecuación } \\
\text { química. }\end{array}$ & $\begin{array}{l}\text { Asigna correctamente los coeficientes } \\
\text { estequiométricos, a partir de los números de } \\
\text { oxidación. }\end{array}$ \\
\hline $\begin{array}{l}\text { Establecer ecuaciones químicas a partir de la información de } \\
\text { productos y reactivos. }\end{array}$ & Escribe correctamente las relaciones estequiométricas. \\
\hline $\begin{array}{l}\text { Identificar en una reacción química de óxido-reducción } \\
\text { (Redox), la sustancia oxidante y la sustancia reductora. }\end{array}$ & $\begin{array}{l}\text { Interpreta adecuadamente la sustancia que se oxida y } \\
\text { la sustancia que se reduce. }\end{array}$ \\
\hline
\end{tabular}

TABLA 3. OBJETIVOS DE APRENDIZAJE Y CRITERIOS DE EVALUACIÓN PARA EL TEMA DE ESTEQUIOMETRIA

\begin{tabular}{l|l}
\multicolumn{1}{c|}{ OBJETIVOS DE APRENDIZAJE } & \multicolumn{1}{c|}{ CRITERIOS } \\
\hline $\begin{array}{l}\text { Identificar las cantidades de una sustancia en mezcla sólidas } \\
\text { y acuosas a través del manejo de diferentes unidades de } \\
\text { concentración. }\end{array}$ & $\begin{array}{l}\text { Interpreta adecuadamente la densidad y la molaridad } \\
\text { como unidad de concentración. }\end{array}$ \\
\hline $\begin{array}{l}\text { Establecer las relaciones estequiométricas entre sustancias } \\
\text { que reaccionan o que se producen, considerando relaciones } \\
\text { molares para identificar reactivo límite y rendimientos de } \\
\text { reacción. }\end{array}$ & $\begin{array}{l}\text { Identifica el reactivo límite y la función que cumple } \\
\text { para la determinación de la cantidad de sustancia que } \\
\text { se produce y/o sustancia en exceso en las reacciones } \\
\text { químicas. }\end{array}$ \\
\hline $\begin{array}{l}\text { Identificar cantidades de sustancias gaseosas que satisfacen } \\
\text { comportamiento ideal. }\end{array}$ & $\begin{array}{l}\text { Determina la cantidad de sustancia de un gas ideal } \\
\text { para establecer relaciones estequiométricas entre } \\
\text { productos y reactivos. }\end{array}$ \\
\hline
\end{tabular}

TABLA 4. OBJETIVOS DE APRENDIZAJE Y CRITERIOS DE EVALUACIÓN PARA EL TEMA DE FÓRMULA QUÍMICA

\section{OBJETIVOS DE APRENDIZAJE}

Identificar las sustancias simples y/o compuestas como fuente de los elementos químicos.

Aplicar la Ley de conservación de la masa.

Determinar la fórmula mínima y/o molecular de una sustancia compuesta.

\section{CRITERIOS}

Determina la cantidad de elemento en una sustancia simple y/o sustancia compuesta.

Determina la cantidad de un elemento después de una trasformación química.

Establece la relación entre las moles de los elementos para determinación de la formula molecular de una sustancia.

TABLA 5. OBJETIVOS DE APRENDIZAJE Y CRITERIOS DE EVALUACIÓN PARA EL TEMA DE EQUILIBRIO QUÍMICO NO ACUOSO

\section{OBJETIVOS DE APRENDIZAJE}

Diferenciar entre equilibrio heterogéneo y homogéneo para determinar la constante de equilibrio.

Identificar la espontaneidad de un sistema en equilibrio a partir de energía libre de Gibbs.

\section{CRITERIOS}

Plantea el equilibrio químico identificando condiciones: iniciales, de reacción y de equilibrio.

Establece la relación de espontaneidad con la energía libre de Gibbs $\Delta \mathrm{G}^{\circ}$ y a su vez con la constante de equilibrio Kp.

Identifica las condiciones de perturbación al momento de calcular las condiciones del nuevo equilibrio.

Reconocer el principio de Le Chatelier como un principio de perturbación de un sistema en equilibrio. 
TABLA 6. OBJETIVOS DE APRENDIZAJE Y CRITERIOS DE EVALUACIÓN PARA EL TEMA DE EQUILIBRIO QUÍMICO ACUOSO

\begin{tabular}{l|l}
\multicolumn{1}{c|}{ OBJETIVOS DE APRENDIZAJE } & \multicolumn{1}{c}{ CRITERIOS } \\
\hline $\begin{array}{l}\text { Determinar el pH de la solución resultante a partir de la } \\
\text { constante de equilibrio de una base y/o un ácido débil. }\end{array}$ & $\begin{array}{l}\text { Plantea las ecuaciones de equilibrio y determina el pH } \\
\text { de la solución. }\end{array}$ \\
\hline $\begin{array}{l}\text { Identificar una solución buffer formada por neutralización de } \\
\text { un ácido y/o base débil. }\end{array}$ & $\begin{array}{l}\text { Reconoce la ecuación Henderson-Hasselbalch, como } \\
\text { una derivación de la ecuación de equilibrio de sistemas } \\
\text { débiles para el cálculo de pH. }\end{array}$ \\
\hline $\begin{array}{l}\text { Identificar una solución buffer formada por adición de ácido } \\
\text { o base conjugada (ion común) a un ácido y/o base débil. }\end{array}$ & $\begin{array}{l}\text { Determina la cantidad en gramos necesarios de la } \\
\text { base y/o el ácido conjugado para la formación de una } \\
\text { solución buffer. }\end{array}$ \\
\hline
\end{tabular}

TABLA 7. OBJETIVOS DE APRENDIZAJE Y CRITERIOS DE EVALUACIÓN PARA EL TEMA DE ELECTROQUÍMICA

\section{OBJETIVOS DE APRENDIZAJE}

Escribir las semirreacciones de una reacción Redox y sus respectivos potenciales de reducción.

Calcular el potencial de la celda a condiciones no estándar empleando la ecuación de Nernst.

Identificar la relación entre el trabajo y el número de electrones aplicando la ley de Faraday.

\section{CRITERIOS}

Plantea la semirreacción del ánodo y la del cátodo para construir una celda electroquímica

Identifica celdas en condiciones estándar y no estándar para determinar su potencial.

Relaciona el trabajo con la ley de Faraday para determinar la masa en gramos de una sustancia que se produce.

\section{Niveles de desempeño}

Para el balanceo de ecuaciones químicas y nomenclatura inorgánica se consideraron dos niveles de desempeño a los cuales se les asigno un porcentaje de $0 \%$ para el menor y $100 \%$ para el mayor. Generalmente dos niveles son considerados como "Correcto" e "incorrecto" (Mertler, 2001). La consideración de solo dos niveles se basa en el hecho de que en química los coeficientes estequiométricos asignados a una ecuación química tipo redox son únicos y en las sustancias química solo se pueden clasificar por su nombre y este debe ser correcto (Tabla 8).

Para el resto de los temas trabajados en el examen parcial y final se consideraron cinco niveles de desempeño teniendo en cuenta "Correcto" e "incorrecto" y se completó después con la redacción de los niveles intermedios. En la Tabla 9 se detallan los niveles de desempeño para el criterio: Determinar la cantidad en gramos de un producto en una reacción química.

TABLA 8. NIVELES DE DESEMPEÑO PARA LOS TEMAS BALANCEO DE ECUACIONES QUÍMICAS Y NOMENCLATURA INORGÁNICA

NIVELES DE DESEMPEÑO

\begin{tabular}{l|l}
\hline \multicolumn{1}{c|}{$\mathbf{0 \%}$} & $\mathbf{1 0 0 \%}$ \\
\hline $\begin{array}{l}\text { Escribe inadecuadamente la estructura de una sustancia, } \\
\text { presentando deficiencias en el uso de la nomenclatura } \\
\text { inorgánica. }\end{array}$ & $\begin{array}{l}\text { Escribe adecuadamente la estructura química de } \\
\text { una sustancia utilizando las correctamente las } \\
\text { nomenclaturas: sistemática, stock y tradicional. }\end{array}$ \\
\hline Plantea de forma inadecuada una ecuación química. & $\begin{array}{l}\text { Escribe correctamente una ecuación química, } \\
\text { identificando reactivos y productos. }\end{array}$
\end{tabular}


TABLA 9. NIVELES DE DESEMPEÑO PARA EL CRITERIO DEL TEMA ESTEQUIOMETRÍA

NIVELES DE DESEMPEÑO

\begin{tabular}{|c|c|c|c|c|}
\hline $0 \%$ & $25 \%$ & $50 \%$ & $75 \%$ & $100 \%$ \\
\hline $\begin{array}{l}\text { No desarrolla } \\
\text { ningún cálculo } \\
\text { ni análisis }\end{array}$ & $\begin{array}{l}\text { No tiene claridad } \\
\text { con el orden de } \\
\text { aplicación de los } \\
\text { conceptos densi- } \\
\text { dad y pureza. }\end{array}$ & $\begin{array}{l}\text { Aplica adecuadamente } \\
\text { el concepto de pureza, } \\
\text { pero no tiene claridad } \\
\text { con la determinación de } \\
\text { la masa de los reactivos } \\
\text { puros. }\end{array}$ & $\begin{array}{l}\text { Aplica adecuadamente } \\
\text { el concepto densidad y } \\
\text { pureza para el cálculo } \\
\text { de los reactivos, pero } \\
\text { no muestra claridad } \\
\text { sobre el procedimiento } \\
\text { para identificar el reac- } \\
\text { tivo límite. }\end{array}$ & $\begin{array}{l}\text { Demuestra comple- } \\
\text { tamente el uso de los } \\
\text { conceptos de densidad y } \\
\text { pureza en las soluciones } \\
\text { acuosas, presentando cla- } \\
\text { ridad conceptual sobre el } \\
\text { procedimiento para iden- } \\
\text { tificar el reactivo límite. }\end{array}$ \\
\hline
\end{tabular}

TABLA 10. RÚBRICA PARA LA EVALUACIÓN DEL CRITERIO: RECONOCE LA NOMENCLATURA INORGÁNICA PARA PLANTEAR LA ESTRUCTURA QUÍMICA DE SUSTANCIAS A PARTIR DEL NOMBRE

\begin{tabular}{l|l|l}
\multirow{2}{*}{ Dimensiones } & \multicolumn{2}{c}{ Niveles de desempeño } \\
\cline { 2 - 3 } & \multicolumn{1}{c|}{$\mathbf{0 \%}$} & \multicolumn{1}{c}{$\mathbf{1 0 0 \%}$} \\
\hline $\begin{array}{l}\text { Propone la estructura química } \\
\text { de una sustancia a partir del } \\
\text { nombre. }\end{array}$ & $\begin{array}{l}\text { Escribe inadecuadamente la estructura } \\
\text { de una sustancia, presentando } \\
\text { deficiencias en el uso de la nomenclatura } \\
\text { inorgánica. }\end{array}$ & $\begin{array}{l}\text { Escribe adecuadamente la estructura } \\
\text { química de una sustancia utilizando } \\
\text { las correctamente las nomenclaturas: } \\
\text { sistemática, tradicional y stock. }\end{array}$ \\
\hline $\begin{array}{l}\text { Plantea adecuadamente una } \\
\text { ecuación química. }\end{array}$ & $\begin{array}{l}\text { Plantea de forma inadecuada una } \\
\text { ecuación química. }\end{array}$ & $\begin{array}{l}\text { Escribe correctamente una ecuación } \\
\text { química, identificando reactivos y } \\
\text { productos. }\end{array}$ \\
\hline
\end{tabular}

\section{Construcción de las rúbricas}

Respecto a los objetivos de aprendizaje planteados a continuación se detallan los ejercicios utilizados para la construcción de las rúbricas.

\section{Nomenclatura inorgánica}

Plantear la ecuación química no balanceada de la reacción entre permanganato de cromo (II), sulfuro de hierro (III) y bicarbonato de potasio para producir: cromato ácido de potasio, monóxido de manganeso, sulfato ácido de potasio, óxido ferroso y anhídrido carbónico.

En la Tabla 10 se detallan las dimensiones y los niveles de desempeño para evaluar el ejercicio de nomenclatura inorgánica.

\section{Estequiometría}

Para la siguiente reacción: $3 \mathrm{Cu}_{(\mathrm{s})}+\mathbf{8} \mathrm{HNO}_{3(\mathrm{ac})}$ $\rightarrow \mathbf{3 C u}\left(\mathrm{NO}_{3}\right)_{2(\mathrm{ac})}+\mathbf{2} \mathrm{NO}_{(\mathrm{g})}+\mathbf{4} \mathrm{H}_{2} \mathbf{O}_{(\mathrm{l})}$. Determinar la masa de nitrato de cobre (II), cuando se ponen a re-

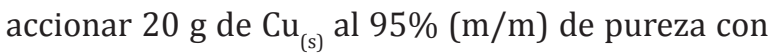
$40 \mathrm{~mL}$ de $\mathrm{HNO}_{3(\mathrm{ac})}$ del $68 \%(\mathrm{~m} / \mathrm{m})$ de pureza y densidad $1,120 \mathrm{~g} / \mathrm{mL}$.

En la Tabla 11 se detallan las dimensiones y los niveles de desempeño para evaluar el ejercicio de estequiometría.

\section{Fórmula química}

El hidrato de cloral $\mathbf{C}_{2} \mathbf{H}_{3} \mathbf{C l}_{3} \mathbf{O}_{2}$ es una sustancia utilizada como hipnótico para sedar a los niños y se suministra en forma de píldora. Antes de ser suministrada, un médico toma una masa de 0,992 g de la píldora que se somete a oxidación completa, generando $0,162 \mathrm{~g}$ de $\mathrm{H}_{2} \mathrm{O}_{(\mathrm{l})}$ y la cantidad de $\mathrm{CO}_{2(\mathrm{~g})}$ con la que se puede obtener exactamente $2,368 \mathrm{~g}$ de $\mathrm{BaCO}_{3(\mathrm{~s})}$. En otro análisis, toma $0,496 \mathrm{~g}$ de píldora y la trata con sodio metálico produciendo $569 \mathrm{~mL}$ de solución de cloruro de sodio $\mathrm{NaCl}_{\text {(ac) }}$ 0,0158 M. A partir del proceso realizado, ¿puede el médico suministrar la píldora a los pacientes? 
TABLA 11. RÚBRICA PARA LA EVALUACIÓN DEL CRITERIO: DETERMINA LA CANTIDAD EN GRAMOS DE UN PRODUCTO EN UNA REACCIÓN QUIIMICA

\begin{tabular}{|c|c|c|c|c|c|}
\hline \multirow{2}{*}{ Dimensiones } & \multicolumn{5}{|c|}{ Niveles de desempeño } \\
\hline & $0 \%$ & $25 \%$ & $50 \%$ & $75 \%$ & $100 \%$ \\
\hline $\begin{array}{l}\text { Identifica el } \\
\text { reactivo límite y } \\
\text { la función que } \\
\text { cumple para la } \\
\text { determinación } \\
\text { de sustancias } \\
\text { que se producen } \\
\text { en las reacciones } \\
\text { químicas }\end{array}$ & $\begin{array}{l}\text { No } \\
\text { desarrolla } \\
\text { ningún } \\
\text { cálculo ni } \\
\text { análisis }\end{array}$ & $\begin{array}{l}\text { No tiene claridad } \\
\text { con el orden } \\
\text { lógico de la } \\
\text { aplicación de } \\
\text { los conceptos } \\
\text { como densidad y } \\
\text { pureza. }\end{array}$ & $\begin{array}{l}\text { Aplica adecuadamente } \\
\text { el concepto de pureza, } \\
\text { pero no tiene claridad } \\
\text { con la determinación de } \\
\text { la masa de los reactivos } \\
\text { puros. }\end{array}$ & $\begin{array}{l}\text { Aplica adecuadamente } \\
\text { el concepto densidad y } \\
\text { pureza para el cálculo } \\
\text { de los reactivos, pero } \\
\text { no muestra claridad } \\
\text { sobre el procedimien- } \\
\text { to para identificar el } \\
\text { reactivo límite. }\end{array}$ & $\begin{array}{l}\text { Demuestra comple- } \\
\text { tamente el uso de los } \\
\text { conceptos de densidad y } \\
\text { pureza en las soluciones } \\
\text { acuosas, con un desarro- } \\
\text { llo aritmético correcto, } \\
\text { presentando claridad } \\
\text { conceptual sobre el pro- } \\
\text { cedimiento para identif- } \\
\text { car el reactivo límite. }\end{array}$ \\
\hline $\begin{array}{l}\text { Establece } \\
\text { relaciones } \\
\text { molares entre el } \\
\text { reactivo límite y } \\
\text { productos. }\end{array}$ & $\begin{array}{l}\text { No } \\
\text { desarrolla } \\
\text { ningún } \\
\text { cálculo ni } \\
\text { análisis. }\end{array}$ & $\begin{array}{l}\text { Muestra rela- } \\
\text { ciones molares } \\
\text { equivocadas, } \\
\text { Sin claridad } \\
\text { en el uso del } \\
\text { rendimiento al } \\
\text { momento de rela- } \\
\text { cionar productos } \\
\text { reales con res- } \\
\text { pecto al producto } \\
\text { teórico. }\end{array}$ & $\begin{array}{l}\text { Establece relaciones } \\
\text { molares adecuadamente } \\
\text { pero con algunas impre- } \\
\text { cisiones como la deter- } \\
\text { minación de la masa } \\
\text { molar, las moles de una } \\
\text { sustancia y el uso del } \\
\text { rendimiento al momen- } \\
\text { to de relacionar produc- } \\
\text { tos reales con respecto } \\
\text { al producto teórico. }\end{array}$ & $\begin{array}{l}\text { Establece relaciones } \\
\text { molares adecuada- } \\
\text { mente con claridad } \\
\text { sobre la determinación } \\
\text { de las moles, pero no } \\
\text { implementa el rendi- } \\
\text { miento para la deter- } \\
\text { minación de la masa } \\
\text { del producto. }\end{array}$ & $\begin{array}{l}\text { Demuestra el dominio de } \\
\text { las relaciones estequio- } \\
\text { métricas, utilizando el } \\
\text { concepto de rendimien- } \\
\text { to y mol, además plantea } \\
\text { un buen desarrollo en } \\
\text { la determinación de la } \\
\text { masa. }\end{array}$ \\
\hline
\end{tabular}

TABLA 12. RÚBRICA PARA LA EVALUACIÓN DEL CRITERIO: DETERMINA LA FORMULA MOLECULAR DE UNA SUSTANCIA PROBLEMA QUE ES SOMETIDA A DESCOMPOSICIÓN

\begin{tabular}{|c|c|c|c|c|c|}
\hline \multirow{2}{*}{ Dimensiones } & \multicolumn{5}{|c|}{ Niveles de desempeño } \\
\hline & $0 \%$ & $25 \%$ & $50 \%$ & $75 \%$ & $100 \%$ \\
\hline $\begin{array}{l}\text { Identifica las } \\
\text { sustancias com- } \\
\text { puestas: } \mathrm{BaCO}_{3 ;} \\
\mathrm{H}_{2} \mathrm{O} \text { y } \mathrm{NaCl}_{\text {, como }} \\
\text { fuente de Carbo- } \\
\text { no, Hidrógeno y } \\
\text { Cloro respectiva- } \\
\text { mente. }\end{array}$ & $\begin{array}{l}\text { No } \\
\text { desarrolla } \\
\text { ningún } \\
\text { cálculo ni } \\
\text { análisis. }\end{array}$ & $\begin{array}{l}\text { No demuestra clari- } \\
\text { dad en la determi- } \\
\text { nación de las moles } \\
\text { de } \mathrm{BaCO}_{3} \mathrm{H}_{2} \mathrm{O}, \mathrm{NaCl} \text {. } \\
\text { Muestra relaciones } \\
\text { molares equivocadas } \\
\text { entre el } \mathrm{C} \text { y el } \mathrm{BaCO}_{3,} \\
\text { entre el } \mathrm{H} \text { y el } \mathrm{H}_{2} \mathrm{O} \\
\text { el } \mathrm{Cl} \text { y el } \mathrm{NaCl} \text {. }\end{array}$ & $\begin{array}{l}\text { Establece relaciones } \\
\text { estequiométricas ade- } \\
\text { cuadamente, pero con } \\
\text { algunas imprecisiones } \\
\text { en la determinación } \\
\text { de las masas de los } \\
\text { elementos y el uso del } \\
\text { concepto de molaridad } \\
\text { al momento de calcular } \\
\text { las moles de } \mathrm{NaCl} \text {. }\end{array}$ & $\begin{array}{l}\text { Establece relaciones es- } \\
\text { tequiométricas adecua- } \\
\text { damente, aunque no } \\
\text { relaciona la molaridad } \\
\text { con la determinación } \\
\text { de las moles de } \mathrm{NaCl} \text {. }\end{array}$ & $\begin{array}{l}\text { Demuestra el } \\
\text { dominio de las } \\
\text { relaciones este- } \\
\text { quiométricas, para } \\
\text { determinar las } \\
\text { moles y la masas } \\
\text { de los elementos C, } \\
\mathrm{HyCl} \text {. }\end{array}$ \\
\hline $\begin{array}{l}\text { Aplica la Ley de } \\
\text { conservación de } \\
\text { la masa. }\end{array}$ & $\begin{array}{l}\text { No } \\
\text { desarrolla } \\
\text { ningún } \\
\text { cálculo ni } \\
\text { análisis. }\end{array}$ & $\begin{array}{l}\text { No tiene claridad en } \\
\text { el proceso de extra- } \\
\text { polación para de- } \\
\text { terminar la cantidad } \\
\text { de un elemento en } \\
\text { diferentes muestras } \\
\text { y no aplica la ley de } \\
\text { la conservación de } \\
\text { la masa. }\end{array}$ & $\begin{array}{l}\text { Presenta algunas impre- } \\
\text { cisiones en la extrapo- } \\
\text { lación de la cantidad de } \\
\text { un elemento en diferen- } \\
\text { tes muestras y aplica- } \\
\text { ción de la ley de conser- } \\
\text { vación de la masa. }\end{array}$ & $\begin{array}{l}\text { Muestra claridad sobre } \\
\text { la extrapolación canti- } \\
\text { dades de sustancia en } \\
\text { diferentes muestras, } \\
\text { pero no aplica la ley } \\
\text { de conservación de la } \\
\text { masa para determinar } \\
\text { la cantidad de oxígeno. }\end{array}$ & $\begin{array}{l}\text { Demuestra domi- } \\
\text { nio en la extrapo- } \\
\text { lación cantidades } \\
\text { de sustancia en } \\
\text { diferentes muestras } \\
\text { y plantea de modo } \\
\text { adecuado la ley de } \\
\text { conservación de la } \\
\text { masa. }\end{array}$ \\
\hline $\begin{array}{l}\text { Determina la fór- } \\
\text { mula mínima y/o } \\
\text { molecular. }\end{array}$ & $\begin{array}{l}\text { No } \\
\text { desarrolla } \\
\text { ningún } \\
\text { cálculo ni } \\
\text { análisis. }\end{array}$ & $\begin{array}{l}\text { No identifica las } \\
\text { relaciones este- } \\
\text { quiométricas, entre } \\
\text { elementos para la } \\
\text { determinación de la } \\
\text { fórmula molecular }\end{array}$ & $\begin{array}{l}\text { Identifica parcial- } \\
\text { mente las relaciones } \\
\text { estequiométricas, entre } \\
\text { elementos, pero no } \\
\text { tiene claridad sobre el } \\
\text { procedimiento para } \\
\text { determinar la fórmula } \\
\text { molecular. }\end{array}$ & $\begin{array}{l}\text { Identifica las relaciones } \\
\text { estequiométricas, entre } \\
\text { elementos, pero no } \\
\text { tiene claridad sobre el } \\
\text { procedimiento para } \\
\text { determinar la fórmula } \\
\text { molecular. }\end{array}$ & $\begin{array}{l}\text { Demuestra el } \\
\text { dominio de las } \\
\text { relaciones este- } \\
\text { quiométricas, y } \\
\text { tiene claridad sobre } \\
\text { el procedimiento } \\
\text { para determinar la } \\
\text { fórmula molecular. }\end{array}$ \\
\hline
\end{tabular}


En la Tabla 12 se detallan las dimensiones y los niveles de desempeño para evaluar el ejercicio de fórmula química.

\section{Equilibrio acuoso}

Determinar el pH de la solución resultante cuando se neutralizan $100 \mathrm{~mL}$ de ácido nitroso 0,500 M con $\mathrm{NaOH}_{\text {(ac) }}$ 0,100 M hasta el punto de equivalencia si a $25{ }^{\circ} \mathrm{C}$ la constante de acidez $K_{a}=$ $4,47 \times 10^{-4}$.

En la Tabla 13 se detallan las dimensiones y los niveles de desempeño para evaluar el ejercicio de equilibrio acuoso.

\section{Equilibrio no acuoso}

$\mathrm{Si}$ a un recipiente de $2 \mathrm{~L}$ se introducen $88,11 \mathrm{~g}$ de $\mathrm{NH}_{4} \mathrm{CN}_{(s)}$ a 298,15 K y cuando la reacción $\mathbf{N H}_{4} \mathbf{C N}_{(s)}$ $\leftrightarrow \mathbf{N H}_{3(\mathrm{~g})}+\mathbf{H C N}_{(\mathrm{g})}$ alcanza el equilibrio, la presión total del sistema es de $1 \mathrm{~atm}$. Calcular las presiones parciales de las sustancias en el nuevo equilibrio, si al recipiente en equilibrio se le retira $\mathrm{HCN}_{(\mathrm{g})}$ hasta reducir a la mitad su presión, manteniendo la temperatura constante.

En la Tabla 14 se detallan las dimensiones y los niveles de desempeño para evaluar el ejercicio de equilibrio no acuoso.

\section{Electroquímica}

Una celda electroquímica galvánica para la producción de oro está compuesta por un ánodo de platino en medio básico y un cátodo de oro. Encontrar el potencial de la celda a $25^{\circ} \mathrm{C}$, si las concentraciones de $\mathrm{SO}_{4}^{2-}(\mathrm{acc})^{2-} \mathrm{SO}_{3}{ }^{2-}(\mathrm{acc})^{\prime} \mathrm{OH}_{(\mathrm{acc})^{-}} \mathrm{AuCl}_{4(\mathrm{ac})^{-}} \mathrm{Cl}_{(\mathrm{ac})^{\prime}}$, son $0,100 \mathrm{M} ; 0,100 \mathrm{M} ; 1 \times 10^{-4} \mathrm{M} ; 0,1 \mathrm{M}$ y $0,4 \mathrm{M}$ respectivamente.

En la Tabla 15 se detallan la dimensión y los niveles de desempeño para evaluar el ejercicio de electroquímica.

TABLA 13. RÚBRICA PARA LA EVALUACIÓN DEL CRITERIO: DETERMINA EL PH DE UNA SOLUCIÓN EN EL PUNTO DE EQUIVALENCIA

\begin{tabular}{|c|c|c|c|c|c|}
\hline \multirow[b]{2}{*}{ Dimensiones } & \multicolumn{5}{|c|}{ Niveles de desempeño } \\
\hline & $0 \%$ & $25 \%$ & $50 \%$ & $75 \%$ & $100 \%$ \\
\hline $\begin{array}{l}\text { Identifica que en } \\
\text { el punto de equi- } \\
\text { valencia ambos } \\
\text { reactivos son el } \\
\text { reactivo límite, y } \\
\text { establece relaciones } \\
\text { a través del manejo } \\
\text { de unidades de } \\
\text { concentración. }\end{array}$ & $\begin{array}{l}\text { No } \\
\text { desarrolla } \\
\text { ningún } \\
\text { cálculo ni } \\
\text { análisis. }\end{array}$ & $\begin{array}{l}\text { No tiene claridad } \\
\text { con el orden lógico } \\
\text { de la aplicación de } \\
\text { las unidades de } \\
\text { concentración y el } \\
\text { concepto de reacti- } \\
\text { vo límite. }\end{array}$ & $\begin{array}{l}\text { Aplica adecuadamente } \\
\text { el concepto de reactivo } \\
\text { límite, pero no tiene } \\
\text { claridad con la deter- } \\
\text { minación de la con- } \\
\text { centración de la base } \\
\text { conjugada. }\end{array}$ & $\begin{array}{l}\text { Aplica adecuada- } \\
\text { mente el concepto } \\
\text { de reactivo límite, } \\
\text { pero presenta difi- } \\
\text { cultad al momento } \\
\text { de determinar la } \\
\text { cantidad de base } \\
\text { conjugada en el } \\
\text { sistema. }\end{array}$ & $\begin{array}{l}\text { Demuestra com- } \\
\text { pletamente el uso } \\
\text { de los conceptos } \\
\text { de reactivo límite, y } \\
\text { unidades de concen- } \\
\text { tración, presentando } \\
\text { claridad conceptual } \\
\text { sobre la determina- } \\
\text { ción de la concen- } \\
\text { tración de la base } \\
\text { conjugada. }\end{array}$ \\
\hline $\begin{array}{l}\text { Determina el pH de } \\
\text { la solución resul- } \\
\text { tante a partir del } \\
\text { equilibrio de la base } \\
\text { conjugada. }\end{array}$ & $\begin{array}{l}\text { No } \\
\text { desarrolla } \\
\text { ningún } \\
\text { cálculo ni } \\
\text { análisis. }\end{array}$ & $\begin{array}{l}\text { Plantea el equilibrio } \\
\text { de neutralización, } \\
\text { pero muestra in- } \\
\text { adecuadamente el } \\
\text { equilibrio de la base. }\end{array}$ & $\begin{array}{l}\text { Establece el equilibrio } \\
\text { de la base conjugada } \\
\text { adecuadamente pero } \\
\text { con algunas imprecisio- } \\
\text { nes como la determi- } \\
\text { nación de la constante } \\
\text { básica } \mathrm{K}_{\mathrm{b}^{\prime}} \text { la concen- } \\
\text { tración de la base con- } \\
\text { jugada y el pH de la } \\
\text { solución resultante. }\end{array}$ & $\begin{array}{l}\text { Establece el equi- } \\
\text { librio de la base } \\
\text { conjugada, pero } \\
\text { presenta dificultad al } \\
\text { momento de correla- } \\
\text { cionar la información } \\
\text { con el pH de la so- } \\
\text { lución. }\end{array}$ & $\begin{array}{l}\text { Demuestra el domi- } \\
\text { nio para establecer } \\
\text { el equilibrio de la } \\
\text { base conjugada, y } \\
\text { el pH de la solución } \\
\text { resultante. }\end{array}$ \\
\hline
\end{tabular}


TABLA 14. RÚBRICA PARA LA EVALUACIÓN DEL CRITERIO: DETERMINA LAS PRESIONES PARCIALES DE LAS SUSTANCIAS EN EL NUEVO EQUILIBRIO

\begin{tabular}{|c|c|c|c|c|c|}
\hline \multirow{2}{*}{ Dimensiones } & \multicolumn{5}{|c|}{ Niveles de desempeño } \\
\hline & $0 \%$ & $25 \%$ & $50 \%$ & $75 \%$ & $100 \%$ \\
\hline $\begin{array}{l}\text { Identifica el principio } \\
\text { de LeChâtelier como } \\
\text { un principio de per- } \\
\text { turbación cuando el } \\
\text { sistema se encuentra } \\
\text { en el equilibrio. }\end{array}$ & $\begin{array}{l}\text { No desarrolla } \\
\text { ningún cálcu- } \\
\text { lo ni análisis. }\end{array}$ & $\begin{array}{l}\text { No identifica con } \\
\text { claridad el princi- } \\
\text { pio de LeChâtelier. }\end{array}$ & $\begin{array}{l}\text { Identifica el princi- } \\
\text { pio de LeChâtelier, } \\
\text { pero con dificulta- } \\
\text { des para interpretar } \\
\text { el concepto de } \\
\text { perturbación. }\end{array}$ & $\begin{array}{l}\text { Identifica el principio } \\
\text { de LeChâtelier pero } \\
\text { muestra imprecisio- } \\
\text { nes en la determina- } \\
\text { ción de las condicio- } \\
\text { nes de perturbación. }\end{array}$ & $\begin{array}{l}\text { Determina con rigor } \\
\text { las condiciones de } \\
\text { perturbación. }\end{array}$ \\
\hline $\begin{array}{l}\text { Redefine las condicio- } \\
\text { nes de la reacción para } \\
\text { determinar las presio- } \\
\text { nes en el nuevo equi- } \\
\text { librio de las sustancias } \\
\text { gaseosas. }\end{array}$ & $\begin{array}{l}\text { No desarrolla } \\
\text { ningún cálcu- } \\
\text { lo ni análisis. }\end{array}$ & $\begin{array}{l}\text { Identifica inade- } \\
\text { cuadamente } \\
\text { las condiciones } \\
\text { iniciales y de } \\
\text { perturbación para } \\
\text { determinar las } \\
\text { condiciones en el } \\
\text { nuevo equilibrio. }\end{array}$ & $\begin{array}{l}\text { Identifica las condi- } \\
\text { ciones iniciales y de } \\
\text { perturbación, pero } \\
\text { muestra imprecisio- } \\
\text { nes conceptuales } \\
\text { sobre el sentido de } \\
\text { desplazamiento de } \\
\text { la reacción. }\end{array}$ & $\begin{array}{l}\text { Establece las condi- } \\
\text { ciones iniciales y de } \\
\text { perturbación, además } \\
\text { establece el desplaza- } \\
\text { miento de la reacción, } \\
\text { pero muestra errores } \\
\text { al momento de deter- } \\
\text { minar las condiciones } \\
\text { en el nuevo equilibrio. }\end{array}$ & $\begin{array}{l}\text { Hace uso adecuado } \\
\text { de los conceptos } \\
\text { de equilibrio para } \\
\text { determinar las con- } \\
\text { diciones en el nuevo } \\
\text { equilibrio. }\end{array}$ \\
\hline
\end{tabular}

TABLA 15. RÚBRICA PARA LA EVALUACIÓN DEL CRITERIO: DETERMINA EL POTENCIAL DE LA CELDA GALVÁNICA A CONDICIONES NO ESTÁNDAR

\begin{tabular}{|l|c|l|l|l|l|}
\multirow{2}{*}{ Dimensiones } & \multicolumn{5}{|c|}{ Niveles de desempeño } \\
\cline { 2 - 6 } & $\mathbf{0 \%}$ & \multicolumn{1}{|c|}{$\mathbf{2 5 \%}$} & \multicolumn{1}{c|}{$\mathbf{5 0 \%}$} & \multicolumn{1}{c|}{$\mathbf{7 5 \%}$} & \multicolumn{1}{|c|}{$\mathbf{1 0 0 \%}$} \\
\hline \multirow{2}{*}{$\begin{array}{l}\text { Calcula el potencial de } \\
\text { la celda a condiciones } \\
\text { no estándar. }\end{array}$} & $\begin{array}{l}\text { No desarrolla } \\
\text { ningún cálcu- } \\
\text { lo ni análisis. }\end{array}$ & $\begin{array}{l}\text { Muestra impre- } \\
\text { cisiones en la } \\
\text { interpretación de } \\
\text { las condiciones } \\
\text { estándary no } \\
\text { estándar. }\end{array}$ & $\begin{array}{l}\text { Interpreta in- } \\
\text { adecuadamente } \\
\text { las condiciones } \\
\text { de la reacción } \\
\text { e interpreta de } \\
\text { modo erróneo el } \\
\text { concepto de celda } \\
\text { galvánica. }\end{array}$ & $\begin{array}{l}\text { ldentifica adecuada- } \\
\text { mente las condiciones } \\
\text { de reacción, pero } \\
\text { muestra imprecisiones } \\
\text { conceptuales en el } \\
\text { cálculo del potencial de } \\
\text { la celda. }\end{array}$ & $\begin{array}{l}\text { Determina adecua- } \\
\text { damente el poten- } \\
\text { cial de una celda } \\
\text { galvánica a condi- } \\
\text { ciones no estándar. }\end{array}$ \\
\hline
\end{tabular}

\section{Implementación de la rúbrica}

La diferencia mínima significativa (LDS) de Fisher fue utilizada metodología para discriminar entre las medias con una confiabilidad del 95\%, encontrando para la metodología "clásica" una diferencia estadística significativa entre los resultados del Examen parcial 2014-2/2015-1 y el Examen final 2014-2/2015-1 con medias de 2,8 $\pm 0,2$ y 2,1 \pm 0,2 respectivamente. Para los resultados de la metodología "nueva propuesta" en la cual se aplicó la rúbrica se reporta una diferencia estadística significativa entre los resultados del Examen parcial 20152/2016-1 y el Examen final 2015-2/2016-1 con medias de 2,9 $\pm 0,2$ y $2,5 \pm 0,2$ respectivamente.

Cuando se compara la implementación de la rúbrica en el examen parcial, la prueba $\mathrm{t}=-0,845$ para un p-valor $=0,399$, indicando que no hay diferencia estadísticamente significativa con un nivel de confianza del 95\% entre las medias de las dos metodologías, pero para la implementación de la rúbrica en el examen final la prueba $t=-3,455$ para un p-valor $=6,12 \times 10^{-4}$, muestra que existe diferencia estadísticamente significativa.

A partir de los métodos de comparación de medianas como la prueba W de Mann-Whitney (Wilcoxon) y la prueba de Kruskal-Wallis con una confiabilidad del $95 \%$ se encontraron diferencias estadísticamente significativas entre el examen parcial y el examen final para metodología "clásica" (W = 11601, estadístico $=34,785$ y p-valor $\left.=3,68 \times 10^{-9}\right)$ y para la metodología "nueva propuesta" ( $\mathrm{W}=11601$, estadístico $=9,477$ p-valor $=2,08 \times 10^{-3}$ ). 
En la Figura 1 se representan los resultados como caja y bigotes y se comparan las metodologías "clásica" (Figura 1a) y "nueva propuesta" (Figura 1b), donde la aplicación de la rúbrica además de disminuir la distancia entre las medianas del examen parcial y final permanece constante el cuartil inferior. El valor extremo en la cola inferior del Examen final 2015-2/2016-1 corresponde a estudiantes que no realizaron el examen.

Para la metodología "clásica" y "nueva propuesta" en el examen parcial, la prueba $\mathrm{W}=-19300$, el estadístico $=0,509$ y $p$-valor $=0,476$, indican que no hay diferencia estadística significativa entre las medianas, con un valor de las medianas igual a 3,0. En el examen final, la prueba $W=22377$, el esta- dístico $=12,500$ y p-valor $=4,08 \times 10^{-4}$ muestra una diferencia estadísticamente significativa entre las medianas (clásica $=2,2$; nueva propuesta $=2,6$ ), por lo cual la aplicación de la rúbrica en el examen final mejoró los resultados de la metodología clásica.

En la Figura 2 se observa la representación de los resultados como la caja y bigotes, para el Examen parcial 2014-2/2015-1 y el Examen parcial 2015-2/2016-1, aunque las medias son iguales, y el cuartil inferir permanece constante, el cuartil superior se encuentra más cercano a 4,0 (Figura 2a). Para el Examen final 2014-2/2015-1 y el Examen final 2015-2/2016-1, se presenta mejoría respecto al cuartil inferior cuando se implementó la rúbrica (Figura 2b).

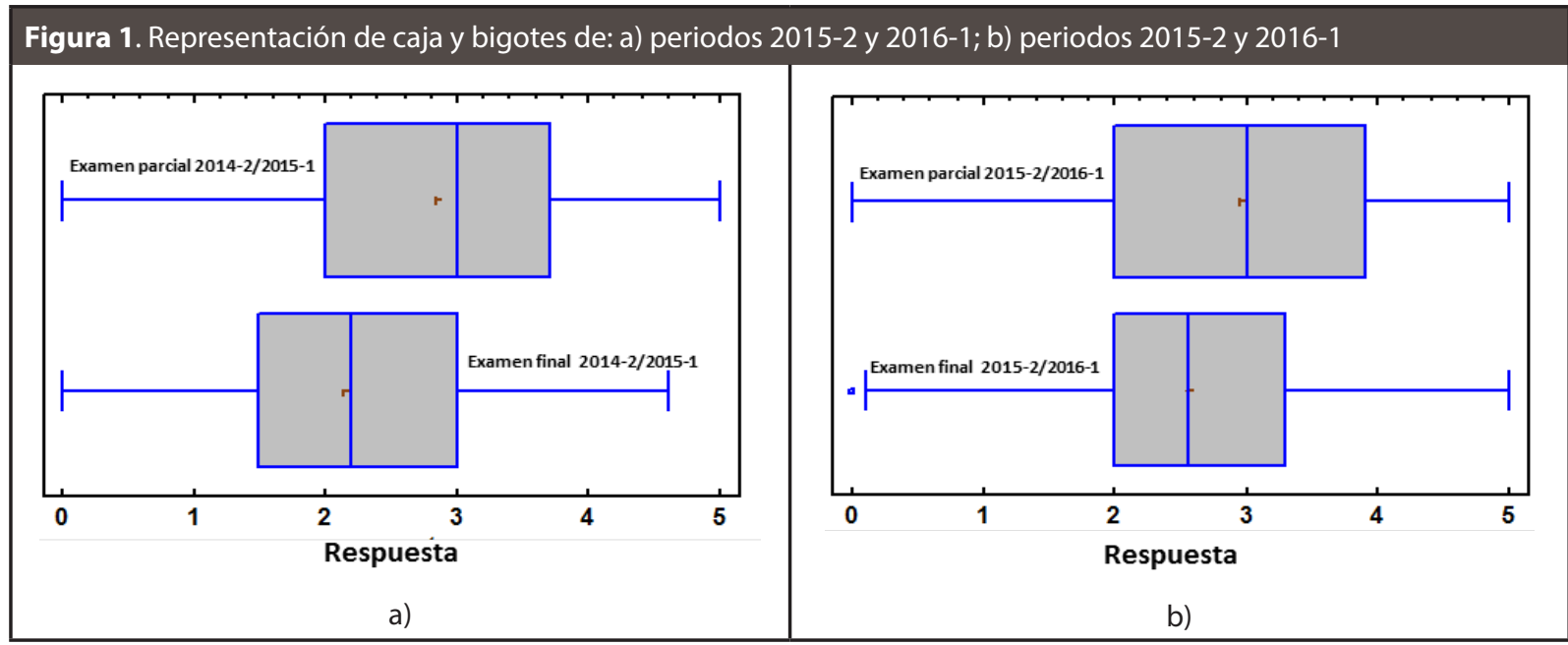

Figura 2. Representación de caja y bigotes de: a) Examen parcial 2014-2/ 2015-1 y 2015-2/ 2016-1; b) Examen final 20142/ 2015-1 y 2015-2/ 2016-1

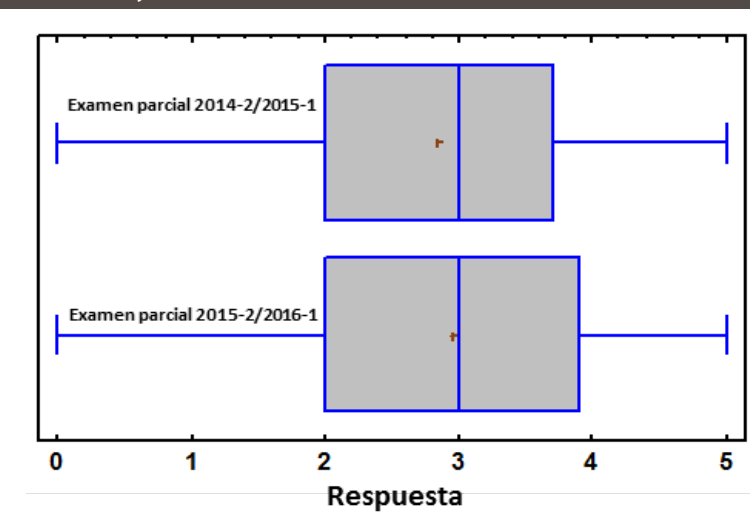

a)

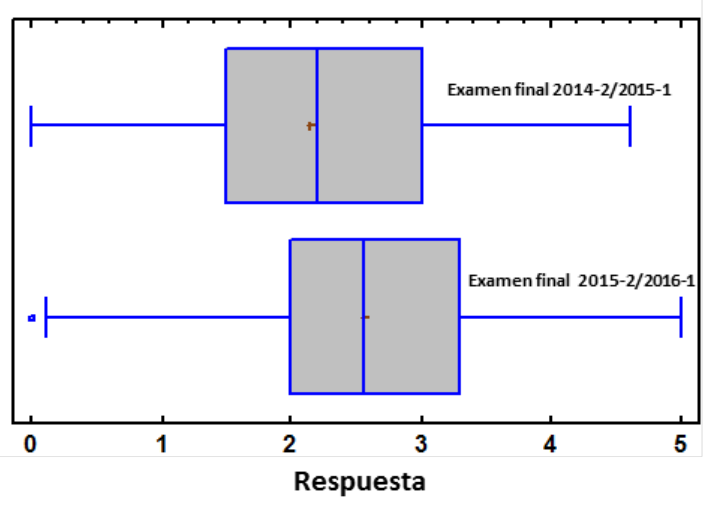

b) 
La implementación de la rúbrica se ve reflejada en el aumento de aprobación del examen parcial y consecuentemente la asignatura como se ilustra en la Tabla 16, en la cual se tiene un incremento del $10 \%$, lo que significa una aprobación de aproximadamente 20 estudiantes.

\begin{tabular}{|c|c|c|c|}
\hline \multirow{2}{*}{ Periodos } & \multicolumn{3}{|c|}{$\begin{array}{l}\text { Porcentaje de estudiantes que } \\
\text { aprueban }\end{array}$} \\
\hline & $\begin{array}{l}\text { Examen } \\
\text { Parcial }\end{array}$ & $\begin{array}{l}\text { Examen } \\
\text { final }\end{array}$ & Asignatura \\
\hline 2014-2/2015-1 & $60 \%$ & $30 \%$ & $70 \%$ \\
\hline $2015-2 / 2016-1$ & $58 \%$ & $42 \%$ & $80 \%$ \\
\hline
\end{tabular}

\section{Conclusiones}

El diseño de las rúbricas lograron establecer características claves para el proceso de implementación como la determinación de los factores que indicarán la calidad del trabajo de los estudiantes en términos de objetivos de aprendizaje y criterios de evaluación, proporcionando una explicación detallada de lo que el estudiante debe realizar para demostrar sus niveles de eficiencia y de esta forma alcanzar los niveles determinados en los objetivos e impulsando la construcción de un sistema de evaluaciones formativas más coherente con las actuaciones de los profesores y estudiantes.

Los estudiantes manifestaron que el proceso de realimentación de la rúbrica les permitió conocer más sobre su fortaleza y debilidades en el proceso de aprendizaje, además que las rúbricas fueron valiosas no sólo como elemento de evaluación sino como instrumento para la reflexión y la comunicación.

Aunque los resultados sobre la aprobación de la asignatura siguen siendo bajos, la implementación de instrumentos de evaluación como la rúbrica debe ser un proceso constante.

La implementación de la rúbrica como instrumento de evaluación nos llama a la reflexión sobre trabajos futuros sobre la investigación e innovación en procesos de enseñanza-aprendizaje y su adaptación al contexto curricular, para proponer actividades en la asignatura y de esta forma potenciar el desarrollar de las competencias pensamiento sistémico, comunicativa y trabajo en equipo.

\section{Referencias}

Araque, P., (2013). Módulo de Química Básica, Medellín, Universidad de Antioquia., pp. 13-18

Bachman, L. F., Palmer, A. S. (1996). Language test development, Language testing in practice. 1 ed. Nueva York, Oxford University Press, pp. 83-133

Cano, M. E., (2008). La evaluación por competencias en la educación superior. Profesorado. Revista de currículum y formación del profesorado, (3),12, pp. 1-16

Gatica L. F., Uribarren B, T. (2013). ¿Cómo elaborar una rúbrica? Investigación en Educación Médica, (1),2, pp. 61-65.

Gipps, C. (1999). Socio-cultural aspects of assessment. Review of Research in Education, 24, pp. 355-392.

Jacob, C., (2001). Analysis and Synthesis, Interdependent operations in chemical language and practice, HYLE-International Journal for Philosophy of Chemistry, (1),7, pp. 31-50

Malini, R. Y., Andrade H. (2010). A review of rubric use in higher education. Assessment \& Evaluation in Higher Education, (4),35, pp. 435-448.

Mansoor, I., Grant, S. (2002). A writing rubric to assess ESL student performance. Adventures in Assessment, 14, pp. 33-38. (ERIC Document Reproduction Service No. ED 482885).

Mertler, C. A., (2001). Designing scoring rubrics for your classroom. Practical Assessment, Research \& Evaluation, (25), 7, pp. 1-9.

Picón E. J., (2013). La rúbrica y la justicia en la evaluación. Íkala, revista de lenguaje y cultura (3),18, pp. 79-94

Shipman D., Roa M., Hooten J., (2012). Using the analytic rubric as an evaluation tool in nursing education: The positive and the negative. Nurse Education Today (3),32, pp 246-249

Torres, J.J., Perera V.H., (2010). La rúbrica como instrumento pedagógico para la tutorización y evaluación de los aprendizajes en el foro online en Educación 
Superior. Pixel-Bit. Revista de Medios y Educación, 36, pp. 141-149

Williams, J. (2003). Preparing to teach writing: Research, theory and practice. 3 ed. Mahwah, Lawrence Erlbaum Associates., pp. 297-331 